\title{
Aplicación de la predicción espacial para el mejoramiento de la cobertura del sistema de bicicletas compartidas en Manizales, Colombia
}

\author{
Juan D. Zuluaga1, Diego A. Escobar ${ }^{1 \star}$ y Carlos A. Moncada ${ }^{2}$ \\ (1) Universidad Nacional de Colombia, Sede Manizales. Facultad de Ingeniería y Arquitectura, Departamento de Ingeniería \\ Civil, Grupo de investigación en Movilidad Sostenible. Campus La Nubia, Carrera 37 - Calle 94, Bloque S2 - oficina 208, \\ Manizales, 170003, Colombia. (correo-e: judzuluagaga@unal.edu.co; daescobarga@unal.edu.co) \\ (2) Universidad Nacional de Colombia, Sede Bogotá. Facultad de Ingeniería, Departamento de Ingeniería Civil y \\ Agrícola, Programa de Investigación en Tránsito y Transporte - PIT. Ciudad Universitaria edificio 214 oficina 417, Bogotá, \\ 111321, Colombia. (correo-e: camoncadaa@unal.edu.co). \\ * Autor a quien debe ser dirigida la correspondencia
}

Recibido Ene. 11, 2021; Aceptado Mar. 13, 2021; Versión final Abr. 14, 2021, Publicado Ago. 2021

\begin{abstract}
Resumen
El objetivo de esta investigación es evaluar la influencia de aumentar el número de estaciones del sistema de bicicletas públicas de Manizales (Colombia) aplicando modelos de oferta del transporte e identificando los sectores beneficiados en términos de tiempo medio de viaje. A partir de la red peatonal, cargada con la velocidad operativa de caminata, se aplican modelos geoestadísticos para el cálculo de las curvas isócronas en tres escenarios, el actual y dos propuestas de nuevas estaciones; luego se realiza un análisis de cobertura geoespacial al relacionar las curvas obtenidas para cada escenario con la capa de información sociodemográfica y de estrato socioeconómico. Ambas propuestas resultaron en un aumento de la cobertura de la población de menor ingreso, contribuyendo a generar mayor equidad. Se concluye que la planeación de la implementación y expansión de los sistemas de bicicletas públicas es crucial para ofertar a un mayor porcentaje de población otras opciones de movilidad eficiente.
\end{abstract}

\section{Application of spatial prediction to improve coverage of the bike-sharing system in Manizales, Colombia}

\begin{abstract}
The primary research objective of the present study is to assess the influence of increasing the number of bike-stations in the bike-sharing systems of the city of Manizales (Colombia) by applying transport supply models and by identifying city areas that would most benefit in terms of average travel time. From the pedestrian network, loaded with the operational walking speed, geo-statistical models are applied for computation of isochronous curves in three scenarios: the current one and two different proposals for new stations. Relating the curves obtained for each scenario to the socio-demographic and socio-economic stratum information layers is analyzed by performing a geospatial coverage analysis. Both proposals resulted in increased coverage of the lower-income population, helping to generate greater equity. It is concluded that planning the implementation and expansion of bike-sharing systems is crucial for offering efficient mobility options to a greater proportion of the population.
\end{abstract}

Keywords: supply transport models; coverage; sustainability; transport systems; urban planning 


\section{INTRODUCCIÓN}

Manizales es la capital de Caldas, reposa sobre la cordillera central colombiana a $2150 \mathrm{msnm}$ en la región centro-occidente de Colombia (Figura 1); se expande en un área urbana de $35,11 \mathrm{~km}^{2}$, habitan 514 mil personas y la red infraestructura de transporte a nivel urbano tiene alrededor de $731 \mathrm{Km}$., existen 74 rutas de transporte público, dos líneas de cable aéreo (4 estaciones) y un sistema de bicicletas compartidas (8 estaciones). En la ciudad se presentan 750 mil viajes diarios, de los cuales el $33 \%$ se realizan en transporte activo, el $29 \%$ en transporte público y el $38 \%$ restante en transporte privado (Alcaldía de Manizales, 2017).

El programa de bicicletas compartidas Ilamado "Manizales en Bici", comenzó en el año 2015 con 3 estaciones y 70 bicicletas disponibles, actualmente el sistema ofrece 211 bicicletas distribuidas en 8 estaciones (Figura 2). Las estaciones actualmente se encuentran en adecuadas condiciones de funcionamiento, se localizan en sectores, en su mayoría, de uso comercial y residencial y poseen capacidades que van de 9 a 20 bicicletas por estación; por otra parte, dada la abrupta topografía de gran parte de la ciudad, las estaciones actuales se localizan en las zonas más altas, a lo largo del filo de la montaña para facilidad de conducción de las bicicletas, no obstante, existe una importante confluencia de modos de transporte lo que dificulta la complementariedad entre éstos, dada la cantidad de rutas de TPCU, vehículos automotores y peatones que circulan por allí. El sistema de cable aéreo permite el transporte de bicicletas, lo cual le ha convertido en un corredor importante de movilidad para las mismas, pues ayuda a superar el accidente natural existente entre el Central Business District (CBD) y el sector de Villamaría.

En esta investigación, la ubicación de las estaciones actuales es evaluada usando un análisis de localización geoespacial. Además, con la información sociodemográfica se establecen comparaciones cuantitativas entre los estratos socioeconómicos y evalúa la inserción de dos propuestas de localización de estaciones de bicicletas, con el objeto de analizar la mejora de cobertura y el desempeño del sistema. La estratificación socioeconómica en Colombia, se estableció a partir de la Ley 142 de 1994, es la forma como se categorizan los hogares mediante criterios basados en las condiciones de la vivienda y el entono donde se encuentra; la estratificación socioeconómica va del estrato 1 al 6 según la capacidad económica del sector, siendo el estrato 1 , sectores con hogares de baja capacidad y el estrato 6, sectores con hogares de alta capacidad (Figura 3).



Fig. 1: Localización geográfica del área de estudio.

Los programas de bicicletas compartidas han existido por 60 años, sin embargo, en la última década han logrado importante popularidad alrededor del mundo como un modo de transporte revolucionario, alternativo 
y sostenible (Shaheen et al., 2010). La operatividad del sistema se basa en proveer estaciones en determinados puntos de la ciudad, permitiendo a las personas acceder a una bicicleta prestada por un breve periodo de tiempo. Se ha establecido que un programa de bicicletas compartidas es eficiente cuando el uso de las mismas es de 4 a 8 veces por día, ya que por debajo de los 4 usos/bicicleta/día existiría un bajo costobeneficio, y por encima de los 8 usos/bicicleta/día, existiría una baja disponibilidad de éstas en las horas pico (Gauthie et al., 2013). El éxito del programa de bicicletas compartidas depende de la provisión en la mayoría de los puntos de acoplamiento de punto de partida y punto de llegada con la cantidad suficiente de bicicletas (Wang et al., 2016), teniendo que una adecuada localización de las estaciones de acoplamiento, mejoraría las condiciones de accesibilidad para usuarios de cualquier nivel socioeconómico (Broach et al., 2012).

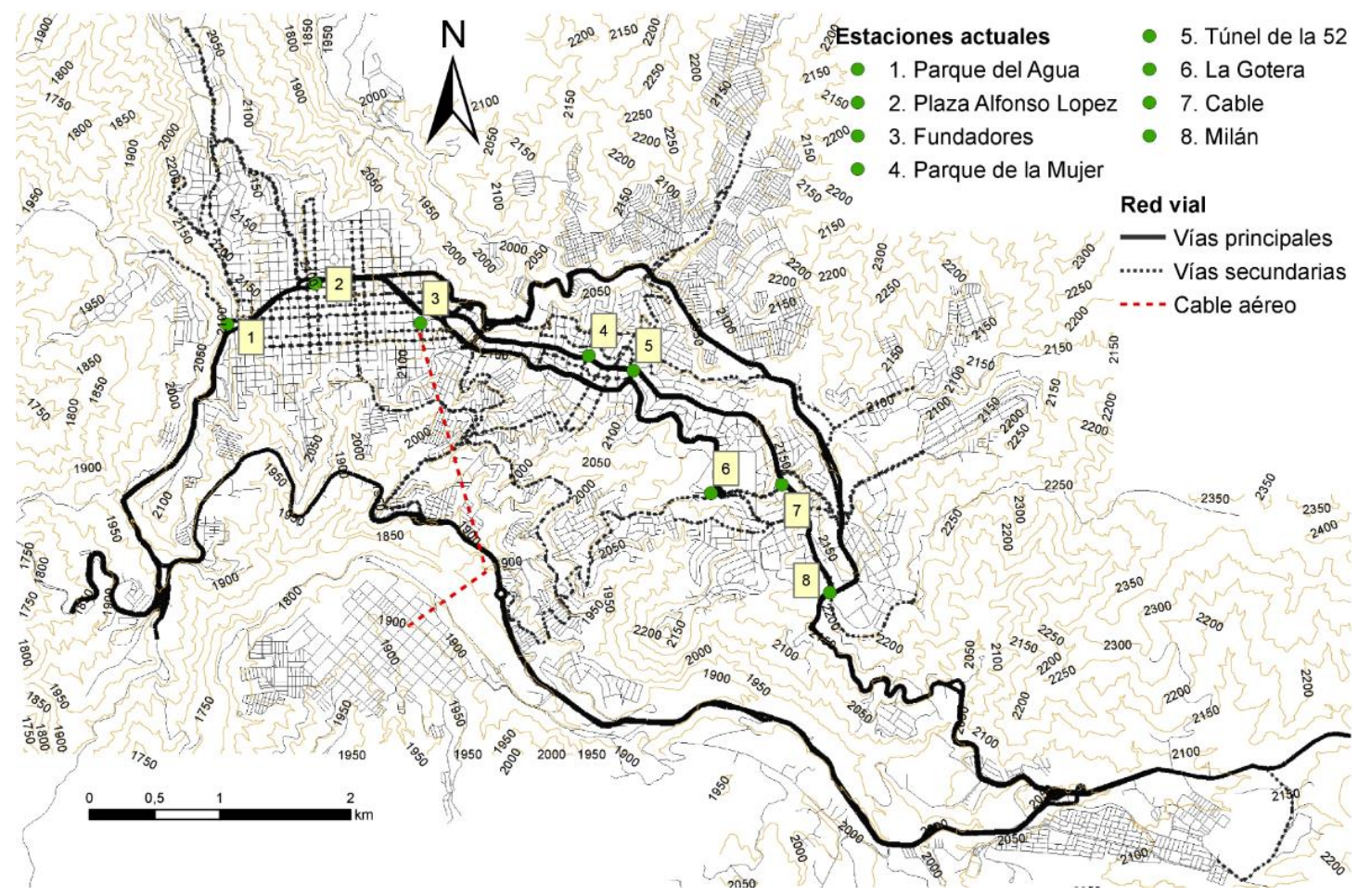

Fig. 2: Localización de las estaciones del sistema de bicicletas compartidas de Manizales. Escenario actual.

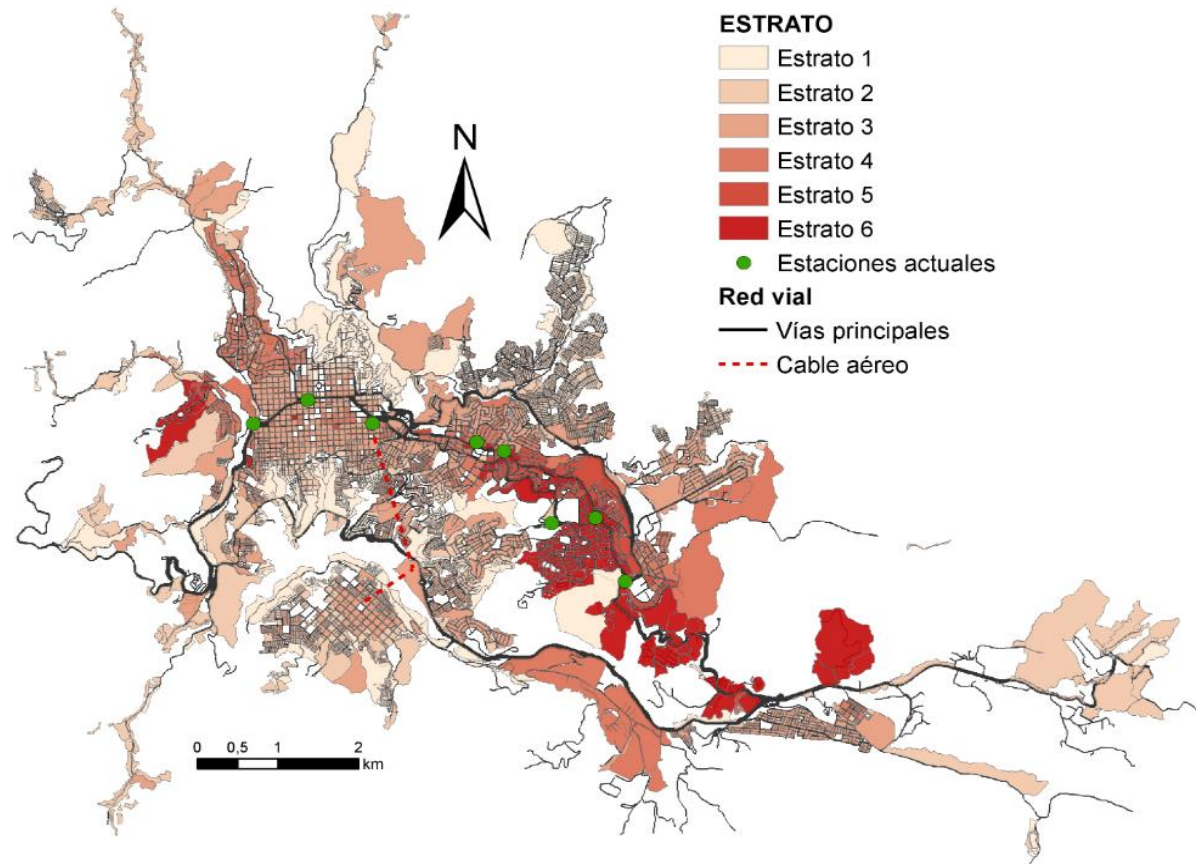

Fig. 3. Estratificación socioeconómica de la zona en estudio.

La definición clásica de accesibilidad se le atribuye a Hansen (1959): "El potencial de las oportunidades para la interacción". Las condiciones de accesibilidad pueden demostrar técnicamente la potencial interacción entre diferentes puntos o zonas geográficas de una ciudad, con el objetivo de identificar la facilidad o la dificultad 
de alcanzar un destino mediante el uso de la infraestructura y los medios de transporte disponibles (Geurs y Van Wee, 2004; Levinson y Wu, 2020). Existen dos tipos de análisis de accesibilidad al considerar la evaluación de todo un sistema: accesibilidad pasiva y activa; la accesibilidad pasiva evalúa cuántos usuarios pueden llegar a cierta ubicación, por ejemplo, la conectividad de cercanía a una estación de bicicletas (García et al., 2012), y una accesibilidad activa describe a cuántos destinos puede llegar la población en un período de tiempo dado usando un modo de transporte. El concepto se ha estudiado en variados campos, como: desplazamientos (Holly, 1993), migración (Kotavaara et al., 2012), flujos de capital (Bröcker et al., 2010) o producción potencial (Gutiérrez et al., 2010).

Otros investigadores han encontrado la relación existente entre las condiciones de accesibilidad y características o índices particulares de un territorio, como: desarrollo económico (Ribeiro y Silva, 2011; Vickerman et al., 1999), indicadores de sostenibilidad (Cheng et al., 2007), usos del suelo (Geurs y Van Wee, 2004; Handy y Niemeier, 1997), distribución del comercio minorista para suministro de bienes menores (Suárez et al., 2012), etc. Así mismo, un análisis de accesibilidad se considera un instrumento de planificación (Papa et al., 2016) que aporta al desarrollo y bienestar de una región (Jiang y Levinson, 2016), lo que ha permitido, por ejemplo, conocer la disparidad existente entre la provisión de infraestructura para transporte privado y público (Benenson et al., 2011; Jaramillo et al., 2012; Salonen y Toivonen, 2013) a partir de análisis comparativos en términos operativos de los modos de transporte (Farber y Grandez, 2017; Sharma et al., 2020). Lo anterior, ha dado soporte para el uso del concepto en la definición de la ubicación óptima de equipos para la prestación del servicio de Bicicletas compartidas (Frade y Ribeiro, 2015; García et al., 2012).

Ahora bien, considerando la perspectiva social, es importante que las personas tengan acceso a diferentes tipos de actividades, que requieren unas adecuadas condiciones de accesibilidad a través de la provisión de sistemas de transporte eficientes y de calidad (Farrington y Farrington, 2005; Martens y Bastiaanssen, 2019). En el caso de los sistemas de bicicletas compartidas, las adecuadas condiciones de accesibilidad a una estación son una variable fundamental para aumentar la probabilidad de que el número de viajes diarios tenga una tendencia creciente al pasar del tiempo (García et al., 2012; Kabra et al., 2019). De acuerdo con la Guía ITDP (Instituto de Políticas de Transporte y Desarrollo) (Gauthie et al., 2013), hay tres factores para valorar una planeación óptima del sistema de bicicletas compartidas: i) La cobertura mínima del sistema de bicicletas compartidas debe ser de $10 \mathrm{~km}^{2}$, resaltando que cada estación influye en un radio de 500 metros a la redonda; ii) La cantidad de bicicletas, se ha apreciado que el valor óptimo de bicicletas es entre 10 y 30 por cada mil habitantes; iii) La densidad de estaciones debe estar entre 10 y 16 estaciones $/ \mathrm{km}^{2}$.

\section{METODOLOGÍA}

La investigación está compuesta por cinco etapas: i) La red de infraestructuras del transporte fue actualizada para la precisión del desarrollo del modelo mientras que la información relacionada con las características operativas de la red fue analizada y determinada; ii) Definición de la velocidad de marcha operativa en la red; iii) Cálculo de la accesibilidad media integral para cada estación de bicicletas desde cualquier punto de la ciudad, en el contexto actual y en dos propuestas; en esta etapa la regla de la guía ITDP es usada, ésta expresa que cada estación influye en 500 metros a la redonda (Gauthie et al., 2013); iv) Cálculo del gradiente porcentual de tiempo medio de viaje ahorrado, dada la inserción de las nuevas propuestas; v) Estimación de los porcentajes de población cubierta por estrato socioeconómico, dadas las curvas isócronas obtenidas.

Acondicionamiento de la malla de transporte y nuevas propuestas: La herramienta principal para desarrollar modelos de accesibilidad es una malla vial de transporte, formada por puntos y arcos; los puntos simbolizan los cruces viales y los arcos los segmentos de vía en donde se carga la velocidad promedio. En algunos casos, se ubican nodos adicionales para mejorar la interpolación de la información. La red de transporte de Manizales fue creada en el Plan Maestro de Movilidad para la ciudad en 2010 (PMM, Plan Maestro de Movilidad para la ciudad de Manizales, 2010) y fue actualizada, basándose en documentos oficiales como la foto aérea de la Alcaldía de Manizales, Google Maps, Street View y Open Street Maps. Manizales en Bici se planeó sin estudios de demanda, y sus estaciones de bicicletas se implantaron de forma intuitiva en lugares donde la gente suele ir a diario. Para la primera propuesta, la previsualización fue la herramienta principal, se identificaron lugares concurridos para definir las nuevas estaciones, proponiéndose un total de 29 estaciones de bicicletas (8 estaciones actuales y 21 estaciones nuevas), aumentando en $262.5 \%$ el número de estaciones en el sistema. Para la segunda propuesta, se utilizaron nuevas estaciones de bicicletas en terrenos residenciales, proponiéndose la inserción de 46 estaciones de bicicletas (8 estaciones actuales y 36 estaciones nuevas), ampliando en un $475 \%$ la cantidad de taquillas en el sistema (Figura 4).

Definición de velocidades operativas: La velocidad operativa se almacenó en los segmentos de vía, ya que provee información para calcular el tiempo de viaje en los arcos. Particularmente, en teoría la velocidad al caminar está definida. Investigaciones anteriores mostraron que el promedio de la velocidad de marcha es de alrededor de $4.32 \mathrm{~km} / \mathrm{h}$, y está relacionada con la pendiente de la red; $0.36 \mathrm{~km} / \mathrm{h}$ para el $10 \%$. 
Estimación de la Accesibilidad integral: La accesibilidad pasiva o integral mide la interconexión entre un nodo particular y la red entera. Un algoritmo del software TransCAD® fue usado para estimar el camino más corto entre un punto en concreto y otros puntos, obteniendo una matriz que contiene el tiempo de desplazamiento mínimo promedio de cada punto. Este algoritmo es conocido como el algoritmo de Dijkstra (Dijkstra, 1959). Después, el vector del tiempo de viaje promedio fue calculado (Ecuación 1). Dónde: $T_{\text {vi }}$ tiempo medio de desplazamiento mínimo entre el punto I y los demás puntos de la malla; tvi= tiempo mínimo de desplazamiento entre el punto I y el punto $\mathrm{J}$; $\mathrm{n}=$ número de puntos en la malla.

$$
T_{v i}=\frac{\sum_{j=1}^{m} t_{v i}}{(n-1)} \quad \mathrm{i}=1,2,3 \ldots ., \mathrm{n} ; \mathrm{j}=1,2,3 \ldots ., \mathrm{m}
$$

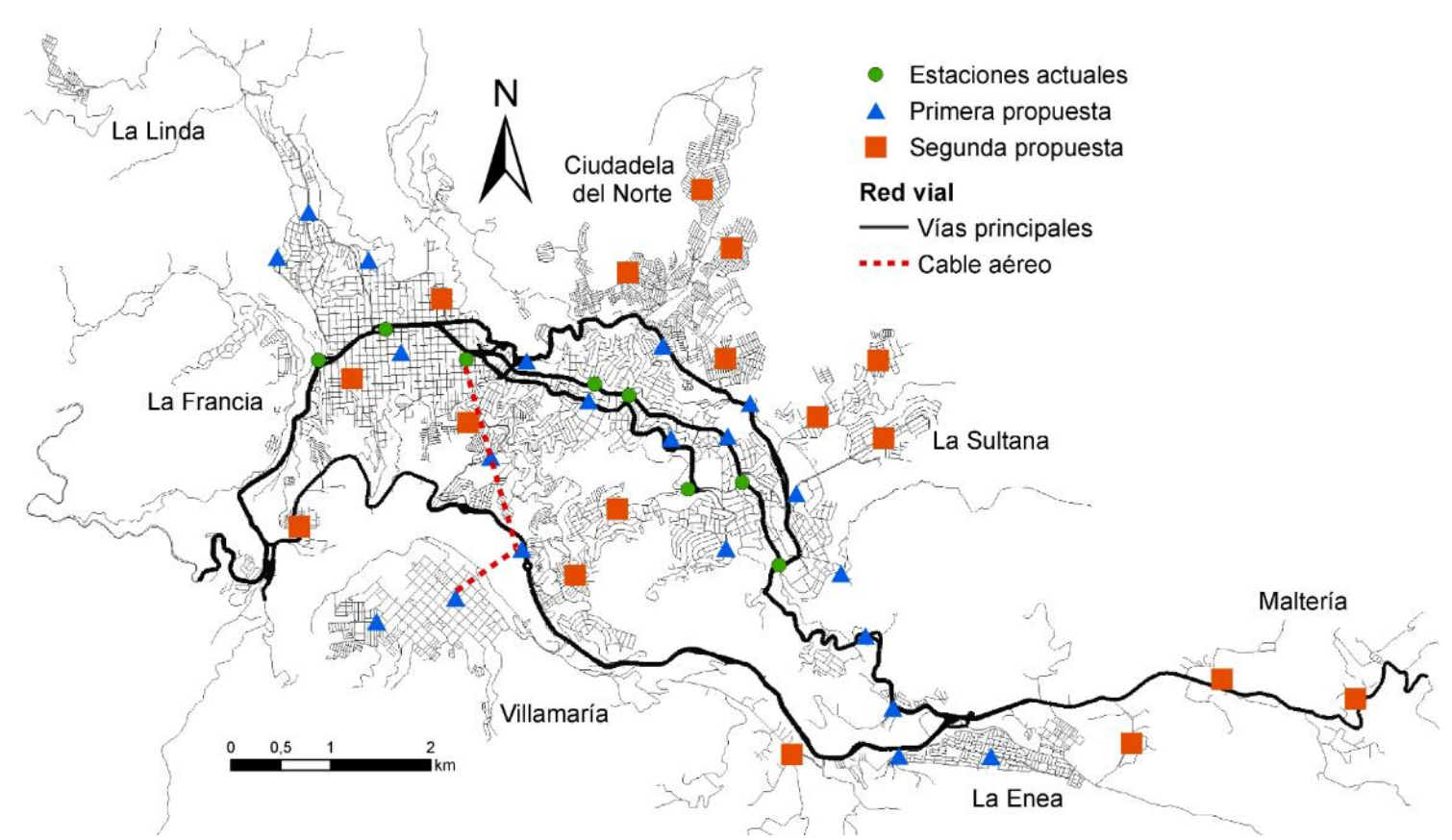

Fig. 4: Localización geográfica de las estaciones de bicicletas. Estaciones actuales, primera y segunda propuesta.

El vector de tiempo de viaje promedio ( $\mathrm{n} \times 1$ ) está asociado con las coordenadas geográficas (latitud y longitud) de cada nodo. Este vector representa las observaciones en el campo, por lo que es esencial crear un modelo para estimar el tiempo de viaje en sectores donde faltan datos de velocidad. El Método Kriging es un método de predicción espacial que incluye autocorrelación, hecho para minimizar el error cuadrático de predicción; el análisis con el método de Kriging considera que la distancia entre los puntos de muestra expresa una correspondencia espacial. Kriging se utilizó con semivariogramas lineales como un prototipo de predicción de tiempo para viajes promedio.

Análisis de cobertura: La quinta y última etapa se ocupa de comparar los resultados de las etapas de previsualización con los datos sociodemográficos proporcionados por las autoridades locales. La ciudad registra 415.124 habitantes distribuidos en 97.666 hogares, con estos datos se hace el análisis de cobertura con las curvas isócronas obtenidas en la cuarta etapa, de tal forma que la ubicación geográfica de las estaciones de bicicletas que se proponen, corresponda con las características sociodemográficas y socioeconómicas de la población.

\section{RESULTADOS Y DISCUSIÓN}

Situación actual: En la Figura 5 se presentan las curvas isócronas de accesibilidad desde las actuales estaciones de bicicletas hasta máximo 500 metros en modo de transporte caminata a través de la red de infraestructura. Se encontró que las personas necesitan invertir entre 0 minutos (color verde) y 13 minutos (color rojo) para llegar a una de las ocho estaciones actuales; en el sector oeste de la ciudad se identifica una mayor área de cobertura que en el sector este, lo anterior debido a la diferencia en términos topográficos de ambos sectores, el primero es ondulado, el segundo es montañoso. En relación con la cobertura espacial de cada estrato, en la Figura 6 se identifican cuatro particularidades: i) el estrato 4, que es el que registra unas mejores condiciones de cobertura espacial y su ojiva porcentual acumulada se encuentra más hacia la izquierda, mostrando que para cubrir el $50 \%$ de su población se deben invertir 5 minutos de tiempo de viaje; ii) el estrato 2, que registra las condiciones de cobertura más deficientes, en donde para cubrir el $50 \%$ de su población se deben invertir hasta 7 minutos de tiempo de viaje; iii) los estratos 3, 5 y 6 que registran la 
necesidad de invertir 6 minutos de tiempo de viaje para cubrir el $50 \%$ de su población; y iv) el estrato 1, que fuera de ser el de menor capacidad económica, no alcanza ninguna de las ocho estaciones del sistema de bicicletas compartidas actuales. Lo anterior, no solo muestra la gran brecha existente en términos de disponibilidad de este sistema de transporte para los estratos de menor capacidad económica, sino que también refuerza la necesidad de expandir de una forma planificada dicho sistema con el fin de mitigar estas condiciones de inequidad identificadas.

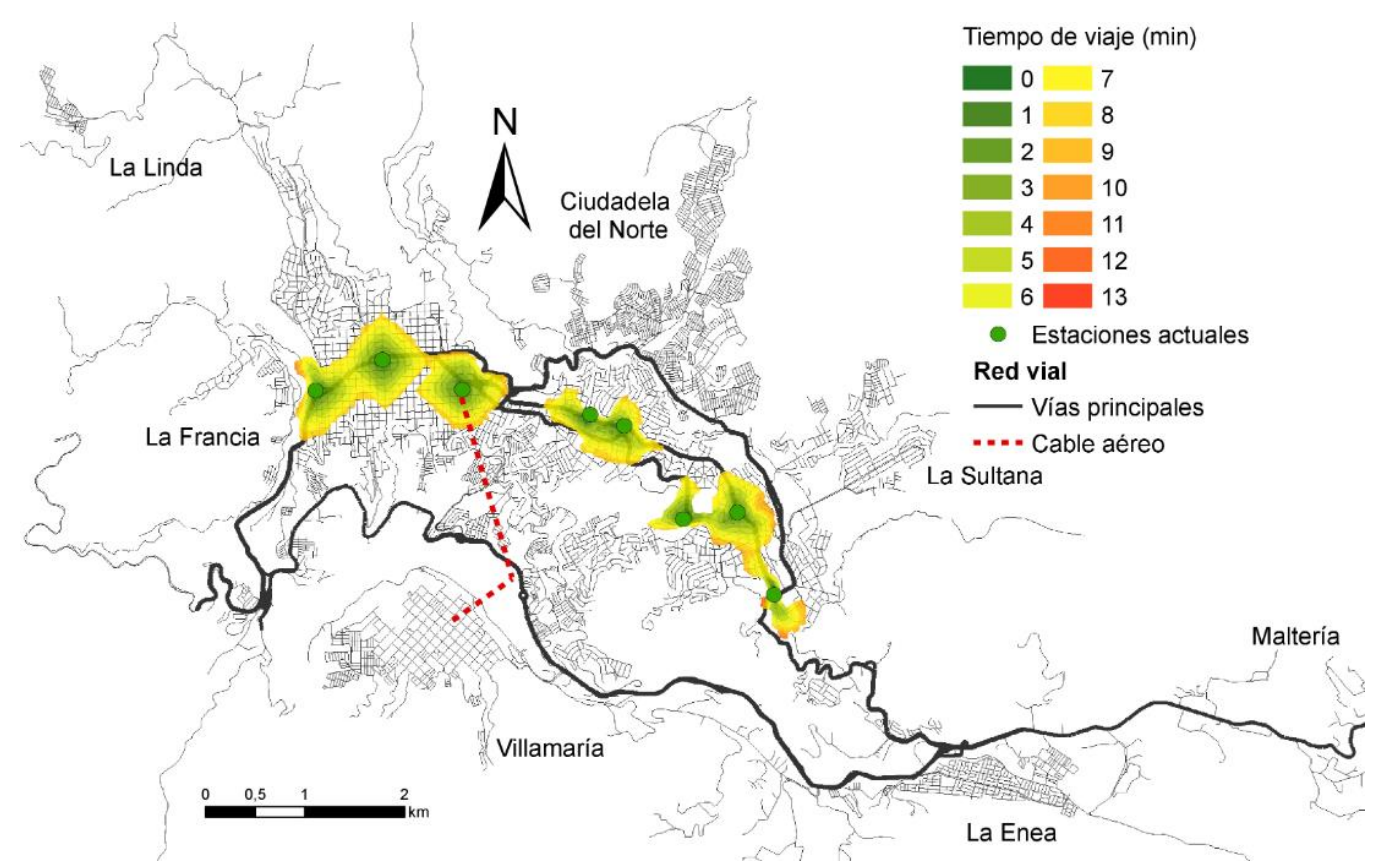

Fig. 5: Curvas isócronas desde las estaciones de bicicletas hasta $500 \mathrm{~m}$. en caminata. Situación actual.

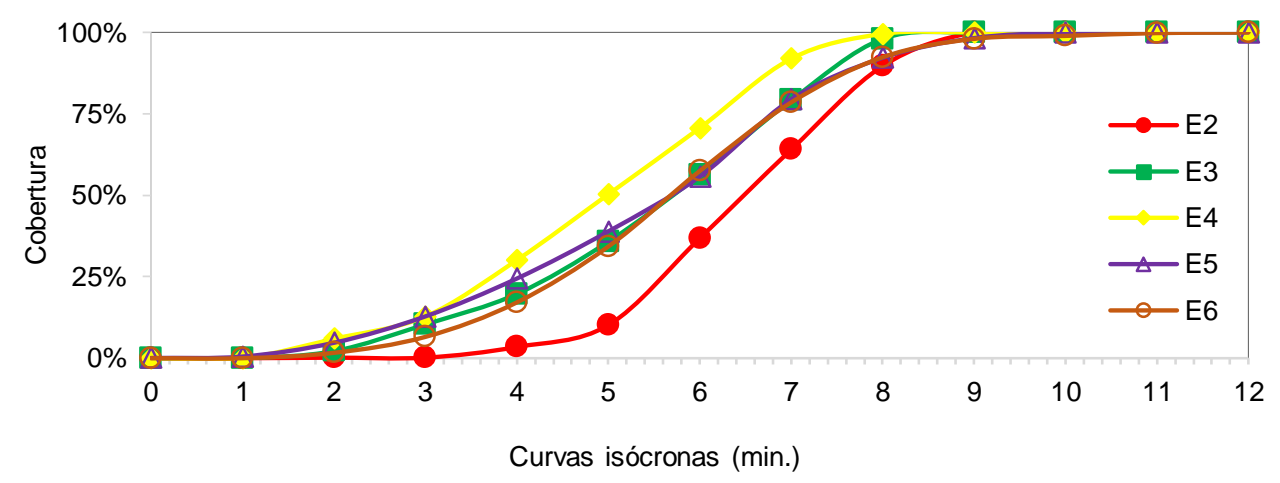

Fig. 6: Ojiva porcentual acumulada de cobertura poblacional según estrato. Situación actual.

Primera propuesta: En la Figura 7 se presentan las curvas isócronas de accesibilidad desde las estaciones de bicicletas que hacen parte de la primera propuesta hasta máximo 500 metros de éstas en modo caminata a través de la red de infraestructura. Se encontró que las personas necesitan invertir entre 0 minutos (color verde) y 20 minutos (color rojo) para alcanzar una de las 29 estaciones (8 actuales y 21 propuestas) que harían parte de esta propuesta. Se propone un aumento del $262 \%$ en relación con el número de estaciones, en donde, a pesar de aumentar el tiempo medio de viaje para llegar a cualquiera de estas estaciones, la cobertura espacial también aumentó y por ende más población podría usar el sistema. Cabe destacar que el cable aéreo proporciona una conexión directa entre estaciones, generando un sistema multimodal que se articula con las actuales rutas de transporte público colectivo urbano. En la Figura 8 se presentan las ojivas porcentuales de cobertura acumulada para cada uno de los estratos socioeconómicos, ésta muestra que el estrato 1 es el de condiciones de cobertura más deficientes, con un valor de 7,9 minutos al ponderar el tiempo de viaje con la población cubierta; al otro extremo se encuentran los estratos altos 5 y 6 , los cuales son los de mejores condiciones de accesibilidad al implementarse la propuesta, pues registran un tiempo de viaje ponderado por población de 5,6 minutos, es decir, 2,3 minutos menos que el estrato bajo. Se observa entonces que el sistema de bicicletas compartidas beneficia a las personas de estratos altos, lo cual reforzaría la actual política de movilidad y transporte de la ciudad, ofreciéndose un modo de transporte alternativo, atractivo para las personas que usualmente usan el automóvil. 
Comparando esta propuesta con la situación actual, se obtienen las curvas gradiente de tiempo medio de viaje que se presentan en la Figura 9; se observa que la localización de las 29 estaciones (8 actuales, 21 propuestas), es decir un aumento del $262 \%$ de estaciones, registran reducción de los tiempos medios de viaje en los lugares donde se ubican las nuevas estaciones, con porcentajes de reducción de hasta el 100\%. Se destaca que con esta propuesta el mayor impacto se presentaría a lo largo del corredor que comunica el sector de ciudadela del norte y la enea (oriente), así mismo, se registran beneficios en el sector de Villamaría (Sur) y en el sector de la Linda (noroccidente).

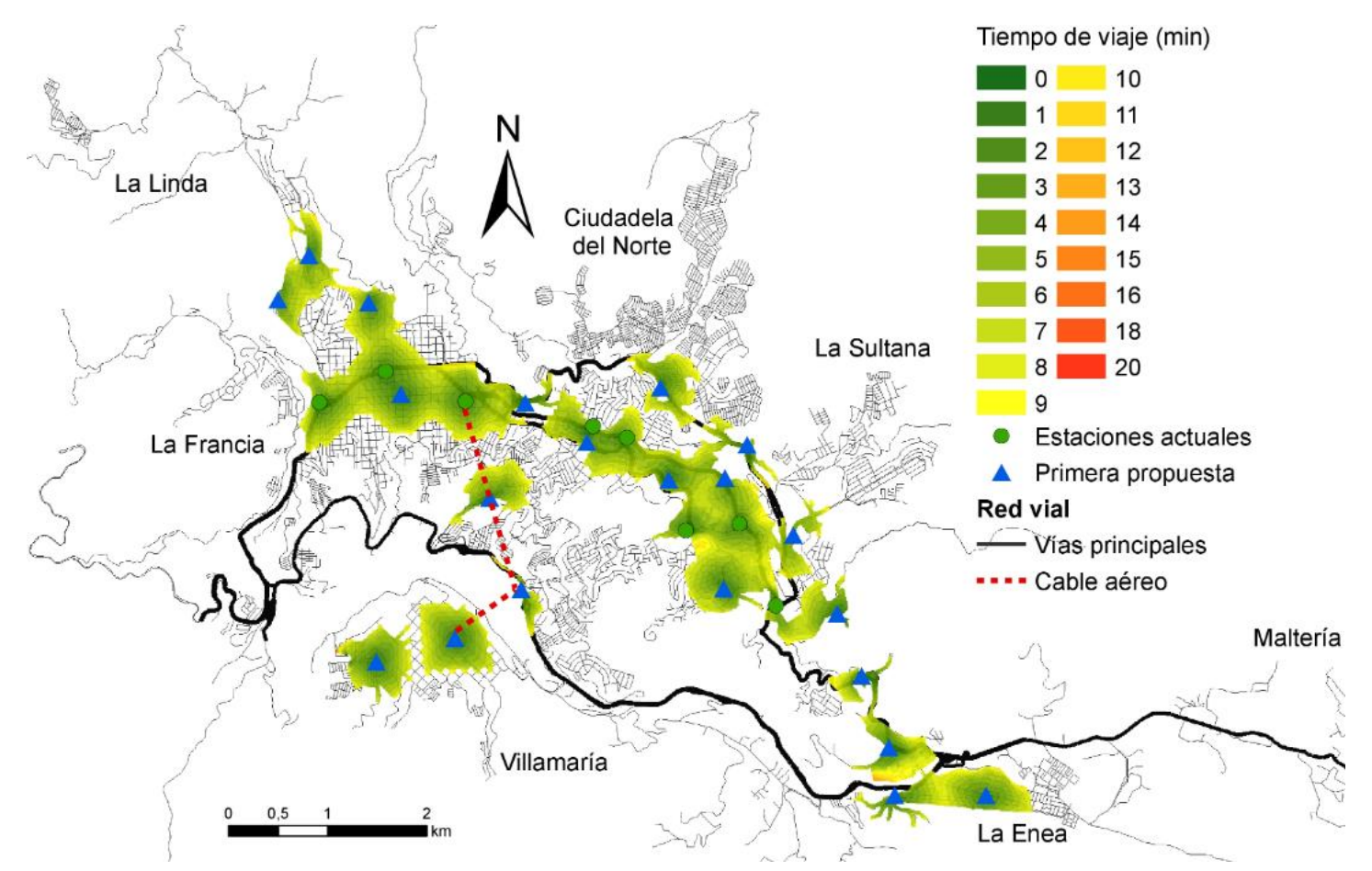

Fig. 7: Curvas isócronas desde las estaciones de bicicletas hasta $500 \mathrm{~m}$. en caminata. Primera propuesta.

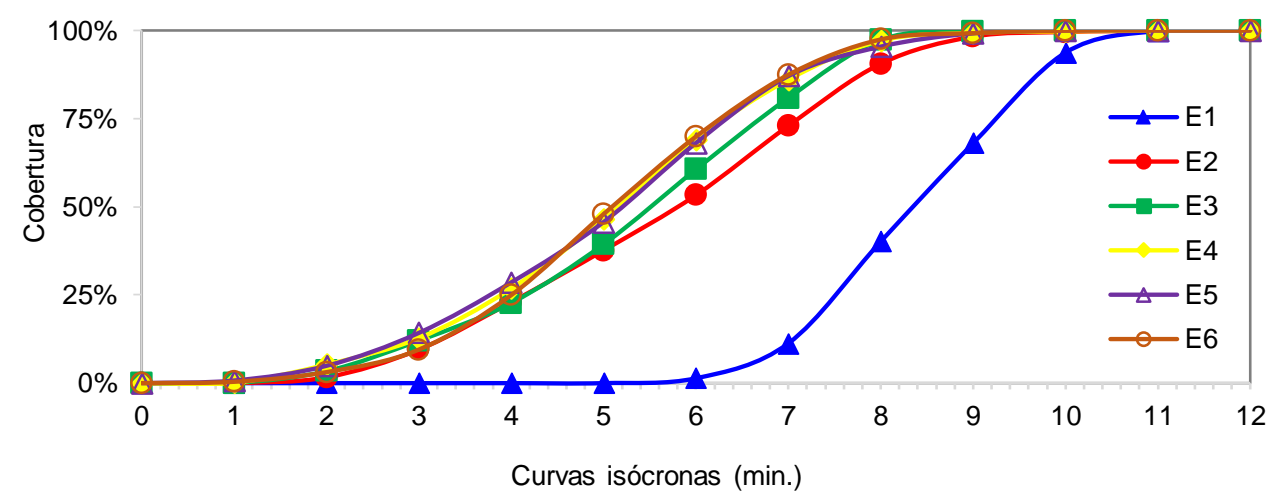

Fig. 8: Ojiva porcentual acumulada de cobertura poblacional según estrato. Primera propuesta.

En la Figura 10, se presenta el porcentaje de ahorro de tiempo de viaje por cada estrato socioeconómico; se identifica que el $90 \%$ de los habitantes de los estratos 1,2 y 4 ahorran el $10 \%$ del tiempo de viaje y los estratos 3,5 y 6 ahorran el $5 \%$. El $50 \%$ de la población muestra casi el $60 \%$ de ahorro en tiempo de viaje para el estrato $4 ; 42 \%$ estrato 2 y $6 ; 35 \%$ estrato 3 y $25 \%$ estrato 1 y 5 . Para el $10 \%$ de los habitantes, el estrato 1 muestra el $60 \%$ de ahorro, el $80 \%$ para el estrato 2 y $5 ; 81 \%$ para el estrato $4 ; 87 \%$ para el estrato 6 ; y $90 \%$ para el estrato 3. En general, se identifica que todos los estratos registran un porcentaje de ahorro, no obstante, se encontró que el estrato 4 es el que registra mayor porcentaje de ahorro de tiempo de viaje, con un $47,2 \%$ de ahorro al ponderar con la población cubierta, mientras que el estrato 1 es el que registra un menor porcentaje de ahorro ponderado por población, llegando al $24,8 \%$.

Segunda propuesta. En la Figura 11 se presentan las curvas isócronas de accesibilidad desde las estaciones de bicicleta que hacen parte de la segunda propuesta hasta máximo 500 metros de éstas en modo caminata a través de la red de infraestructura. Las personas necesitan invertir entre 0 minutos (color verde) y 17 minutos (color rojo) para llegar a cualquiera de las 46 estaciones de bicicletas (8 actuales, 21 primera propuesta, más 17 segunda propuesta). Se propone entonces un aumento del $575 \%$ en relación con el número de estaciones 
de la situación actual. La propuesta se enfoca en proveer acceso al sistema de bicicletas compartidas cerca de usos del suelo residencial, incluyendo zonas periféricas de la ciudad. En la Figura 12, se analiza la cobertura de cada estrato, mostrando una mejora en la equidad entre éstos; el estrato 1 necesita invertir 6,6 minutos para abarcar el $50 \%$ de la población, mientras que, para el resto, es necesario invertir cerca de 5 minutos para abarcar el $50 \%$ de la población; la diferencia entre el estrato 1 y el resto es superior a 1,5 minutos, que a pesar de ser un valor de tiempo bajo, muestra que la brecha entre estratos disminuyó, para el mismo porcentaje de cobertura, en más de 1,5 minutos al comparar con el resultado encontrado en la primera propuesta (figura 8). Comparando los tiempos medios de viaje ponderados por población, es posible fortalecer este resultado de reducción de brecha, pues en la primera propuesta la diferencia entre el estrato 1 y el estrato 6 era de 2,3 minutos, mientras que, con la implementación de la segunda propuesta, esta diferencia es muchas menor y llega a ser de tan solo 0,3 minutos.

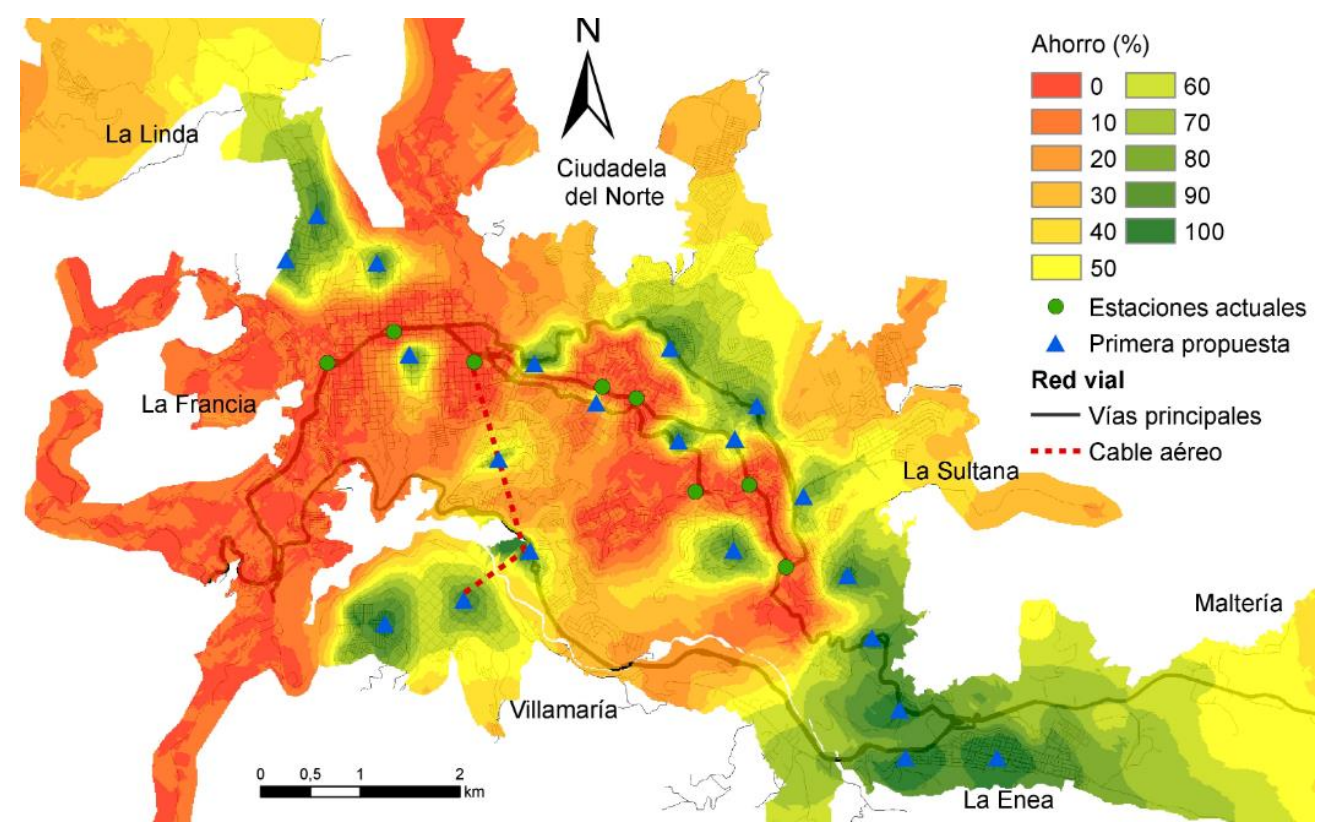

Fig. 9: Gradiente de tiempo medio de viaje. Primera propuesta Vs Situación actual.

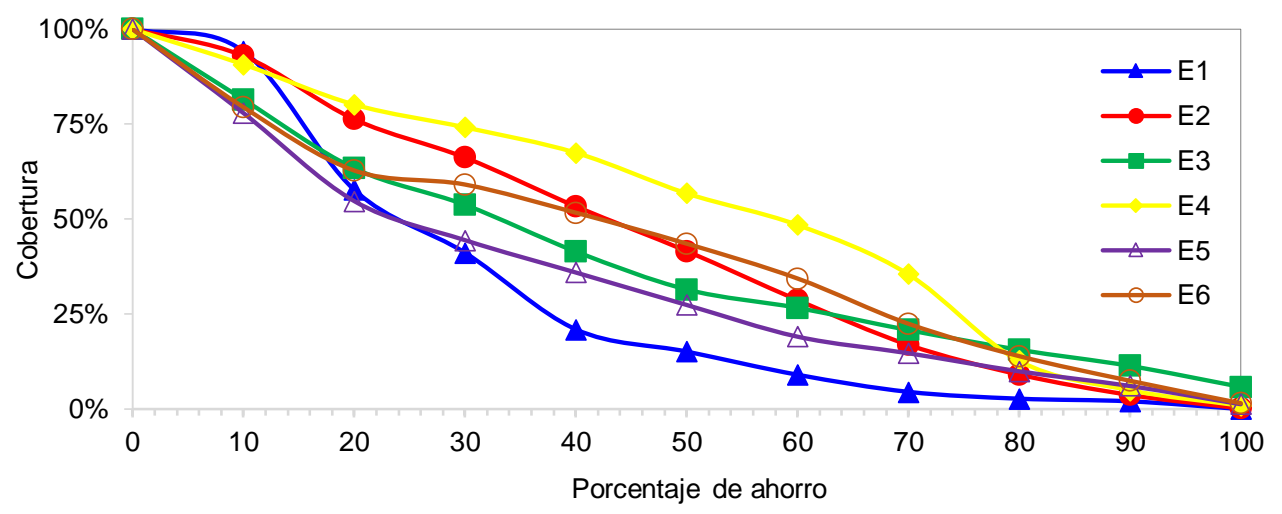

Fig. 10: Porcentaje de ahorro por estrato socioeconómico. Primera propuesta.

Al comparar esta propuesta con la situación actual, en la Figura 13 se presentan las curvas gradiente de tiempo medio de viaje, identificándose que los lugares donde se ubican las nuevas estaciones, registran ahorros de hasta el $100 \%$, mejorándose el acceso en dos sectores, el noroccidente y el suroriente. En la Figura 14, se muestra el porcentaje de ahorro de tiempo de viaje por cada estrato socioeconómico, encontrándose que para el $90 \%$ de los habitantes, el estrato 2 ahorra el $35 \%$ del tiempo de viaje; $30 \%$ para el estrato 1; y más del $10 \%$ para los estratos 3 y 4, mientras que en la primera propuesta los estratos 1 , 2 y 4 mostraron un $10 \%$ de ahorro en términos de tiempo de viaje y un $5 \%$ para el estrato 3; para el $50 \%$ de los habitantes, el estrato 4 ahorra casi el $70 \%$, cuando en la primera propuesta mostró el $60 \%$; $75 \%$ para el estrato 2, mientras que en la primera propuesta mostró $42 \%$; $65 \%$ para el estrato 3, cuando se ahorró el $35 \%$ en la primera propuesta y casi el $50 \%$ para el estrato 1, mientras que en la primera propuesta mostró el $25 \%$. En general, los estratos de bajos y medianos ingresos mostraron un aumento en los ahorros de tiempo medio de viaje, mientras que para los estratos altos el porcentaje de ahorro se mantuvo estable. 


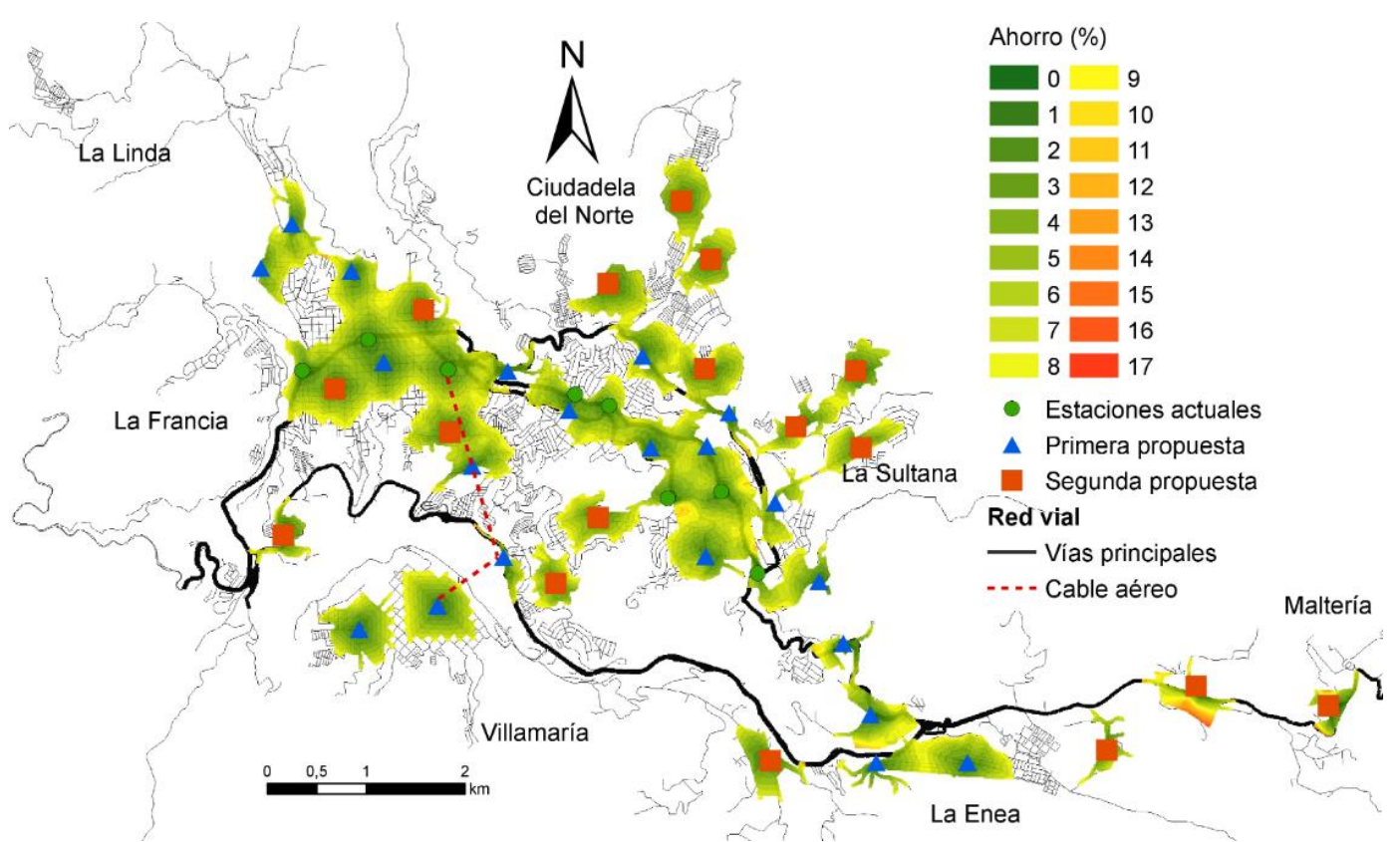

Fig. 11: Curvas isócronas desde las estaciones de bicicletas hasta $500 \mathrm{~m}$. en caminata. Segunda propuesta.

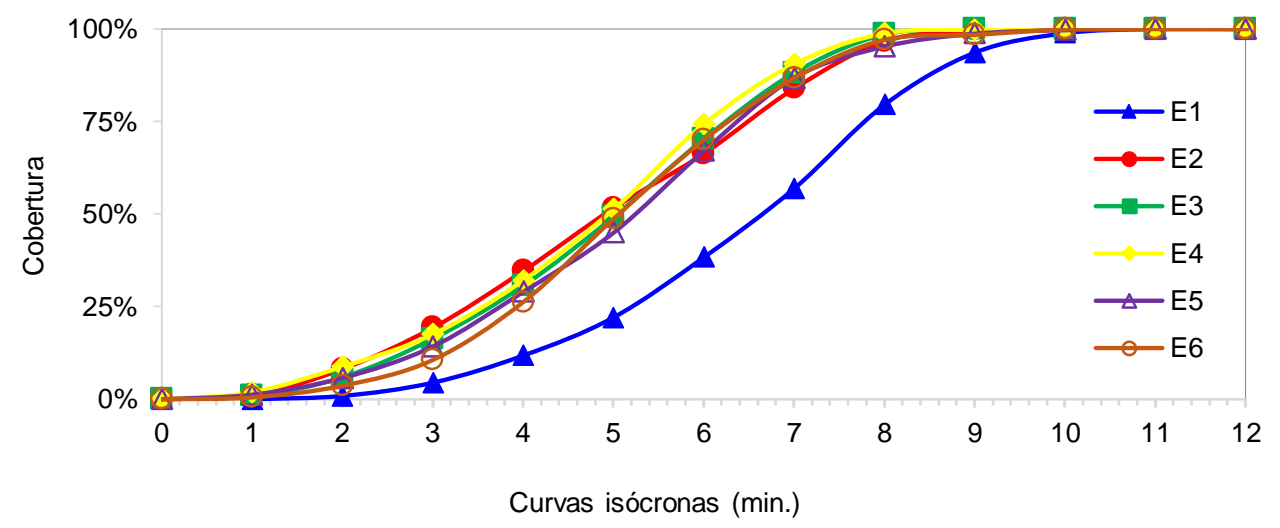

Fig 12: Ojiva porcentual acumulada de cobertura poblacional según estrato. Segunda propuesta.

Comparación de las propuestas con la situación actual. En la figura 15a se presentan las ojivas porcentuales de cobertura acumulada para la situación actual y para cada una de las dos propuestas de intervención del sistema de bicicletas compartidas. Calculando el tiempo medio de viaje ponderado por población, se obtuvo un valor de 5,9 minutos para la situación actual, el cual disminuye a 5,8 minutos para la primera propuesta y a 4,4 minutos para la segunda propuesta; así mismo, se encontró que para cubrir el $50 \%$ de la población que se localiza dentro de los 500 metros de influencia (Gauthie et al., 2013), se necesita invertir un promedio de 5 minutos en la segunda propuesta, mientras que, en la primera propuesta y el contexto actual, las personas deben gastar 5,4 y 5,6 minutos, respectivamente. Se obtienen mejores valores de cobertura acumulada mientras la ojiva se ubique más hacia la izquierda y refiera una mayor pendiente, se tiene entonces que ambas propuestas están a la izquierda de la curva de cobertura de la situación actual, indicando que ambas propuestas refieren algún tipo de mejora en dicha cobertura; no obstante, al comparar las curvas de ambas propuestas, se identifica que la curva de situación actual y la de la primera propuesta convergen para las curvas isócronas de menor y mayor valor, lo cual deje en claro que la segunda provee mayores beneficios de cobertura y acceso a la población. Por otra parte, en la figura 15b se presenta la comparación de las curvas de porcentaje de ahorro en cobertura de la población entre propuestas, en ésta se observa que el $90 \%$ de los habitantes tienen al menos el $10 \%$ de ahorro en la primera propuesta, mientras que, en la segunda propuesta, tiene al menos el $15 \%$ de ahorro; el $50 \%$ de la población tiene al menos un $40 \%$ y un $67 \%$ de ahorro en la primera y segunda propuestas, respectivamente; y el $10 \%$ de la población tiene al menos el $80 \%$ de ahorro en la primera propuesta, mientras que, en la segunda, la población tiene alrededor del 100\% de ahorro en tiempo de viaje. 


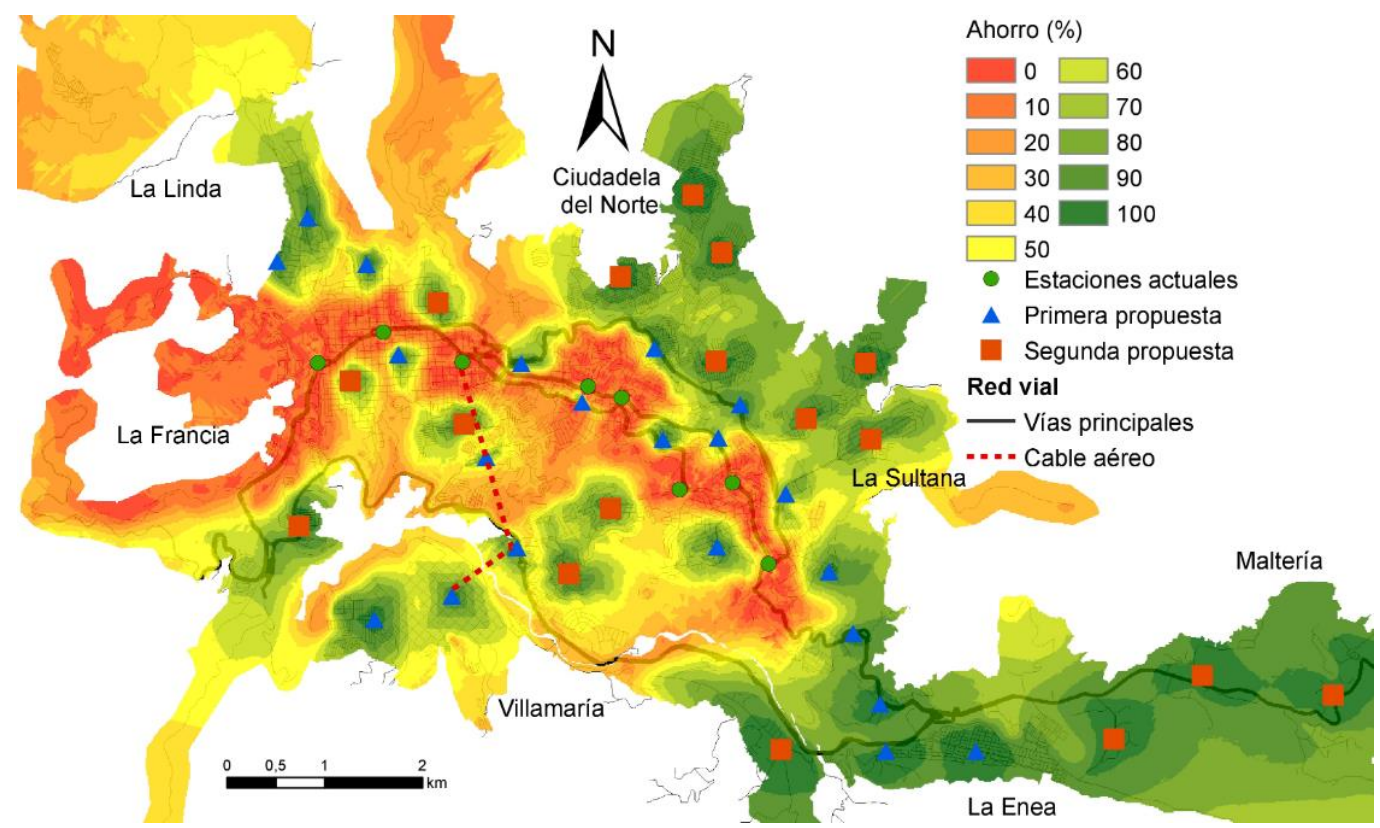

Fig. 13: Gradiente de tiempo medio de viaje. Segunda propuesta Vs Situación actual.

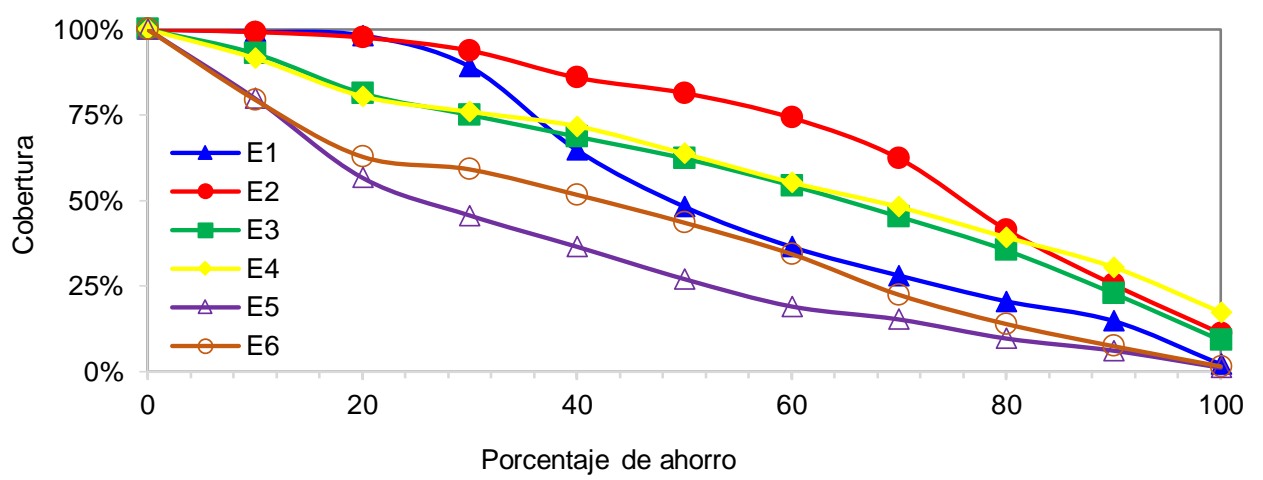

Fig. 14: Porcentaje de ahorro por estrato socioeconómico. Segunda propuesta.
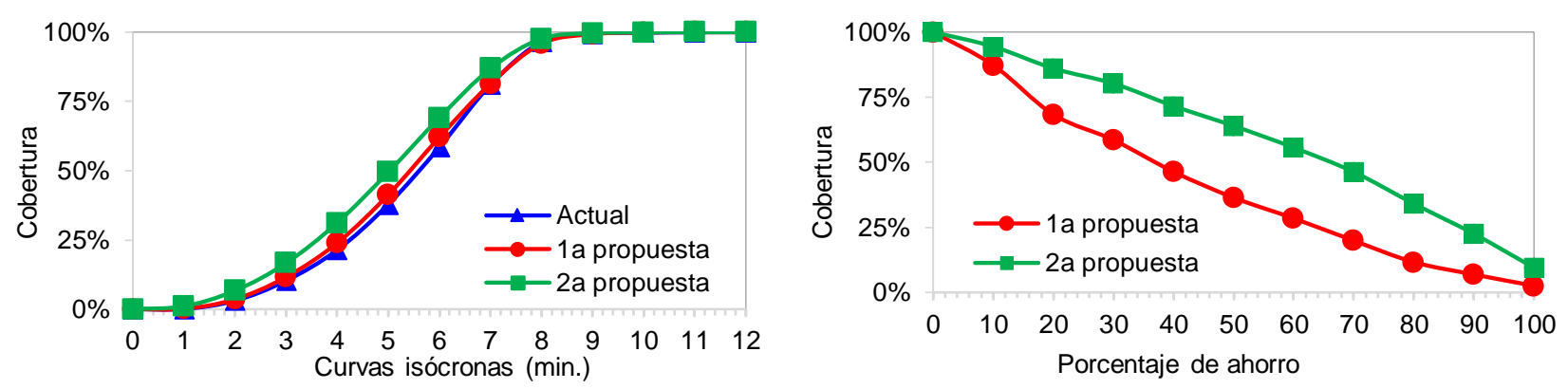

Fig. 15: a) Ojiva porcentual de cobertura acumulada de la población, situación actual y las dos propuestas, b) comparación de porcentaje de ahorro en cobertura de la población entre propuestas.

Ahora bien, al calcular el porcentaje de ahorro ponderado por población, se tiene que la primera propuesta proveería un ahorro del 36,6\% en relación con la situación actual, mientras que la segunda propuesta registra un 56,4\% de ahorro del tiempo medio de viaje en relación con la situación actual. Estos resultados refuerzan la conclusión de que la segunda propuesta tiene más beneficios que la primera al aumentar el número de estaciones, aumentar el número de orígenes y destinos para las personas y reducir el tiempo de acceso a las estaciones. Por otra parte, en la Figura 16 se comparan las curvas de cobertura porcentual de habitantes versus porcentaje de ahorro en tiempo de viaje, según estrato socioeconómico. Mientras más divergentes sean las curvas entre propuestas para un mismo estrato, significa un mayor impacto; teniendo en cuenta lo anterior, los estratos en los que se observa una mayor separación de las curvas es en los estratos 1 y 2 (bajos), mientras que en los estratos 5 y 6 (altos), dicha separación es mínima. Así mismo, al comparar los porcentajes de ahorro de tiempo de viaje ponderado por población según estrato socioeconómico, se encontró que la diferencia entre ambas propuestas de intervención, para los estratos 1 y 2 (bajos) son de 25,4\% y 
$28,4 \%$, mientras que en los estratos 5 y 6 (altos), dicha diferencia es de $0,5 \%$ y $1,4 \%$, respectivamente. Lo anterior indica que para los estratos altos es indiferente si se implementa la primera o la segunda propuesta, pero para los estratos bajos, la segunda propuesta es ampliamente más beneficiosa.

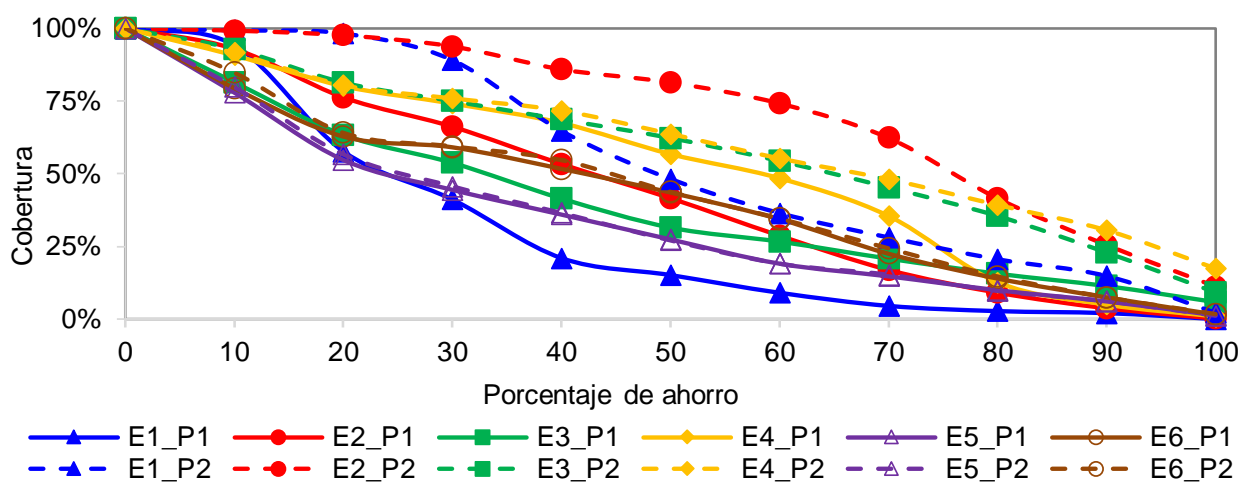

Fig. 16: Comparación de habitantes cubiertos por las propuestas Vs porcentaje de ahorro en tiempo de viaje.

\section{CONCLUSIONES}

Las políticas de transporte actuales en los países en desarrollo han basado su movilidad en el automóvil como su modo principal de transporte, lo que ha contribuido a que las principales externalidades negativas como la congestión, la contaminación y la accidentalidad aumenten; por su parte, los sistemas de bicicletas compartidas se han convertido en un modo de transporte alternativo, sostenible y óptimo para superar dichas externalidades y minimizar su impacto en la población y en el medio ambiente. El objetivo principal de mejorar las condiciones de acceso al sistema se logra al aumentar el número de estaciones de bicicletas, pues amplía los pares de OyD que se pueden cubrir, teniendo que los modelos de accesibilidad son un instrumento de planificación potente para demostrar la variabilidad en la cobertura geoespacial y analizar los impactos positivos que podrían mejorar la calidad de vida de la población. Los resultados de esta investigación indican que aumentar el número de estaciones podría mejorar el rendimiento del sistema de bicicletas compartidas, el cual puede planificarse con el fin de generar mayor beneficio a la población. Se encuentra que las abruptas condiciones topográficas en algunos sectores de la ciudad, sumada a la falta de estaciones de bicicletas públicas en zonas residenciales, hace que las personas decidan utilizar otros modos de transporte para realizar los viajes cotidianos, lo cual es un obstáculo para establecer el sistema como una opción diferente a los modos motorizados, siendo necesario el incluir e impulsar la posibilidad de uso de bicicletas electroasistidas. Sin embargo, debe modelarse el comportamiento de la demanda para optimizar la ubicación de nuevas estaciones futuras y atender con mayor precisión la demanda inducida que se genere en el sistema. El hecho de que los datos de demanda no estén disponibles indica que la autoridad de transporte debe complementar la presente investigación para fortalecer técnicamente los resultados obtenidos y satisfacer las necesidades de movilidad de la población, involucrando la variable clima mediante la aplicación de encuestas de preferencia modal declarada. La metodología aplicada en la presente investigación, sirve de instrumento técnico para cualquier entidad territorial que busque comprender los impactos que, sobre las dinámicas de movilidad, imprimen este tipo de proyectos sobre su territorio, siendo necesaria información sociodemográfica y socioeconómica que comúnmente poseen los funcionarios y técnicos de dichas entidades.

\section{AGRADECIMIENTOS}

Esta investigación se realizó a través del anuncio nacional de proyectos para el fortalecimiento de la investigación, creación e innovación de la Universidad Nacional de Colombia 2016-2018, Código: 35178. Los autores desean agradecer al Dirección de Investigaciones por apoyar a los jóvenes a desarrollar estudios. Se agradece a los pares evaluadores por las contribuciones realizadas.

\section{REFERENCIAS}

Alcaldía de Manizales, Plan Maestro de Movilidad de Manizales. Financiera de Desarrollo Territorial SA, Steer Davies Glave, Manizales (2017)

Benenson, I., Martens, K., Rofé, Y., y Kwartler, K., Public transport versus private car gis-based estimation of accessibility applied to the Tel Aviv Metropolitan Area, https://doi.org/10.1007/s00168-010-0392-6, Annals of Regional Science, 47, 499-515 (2011)

Broach, J., Dill, J., y Gliebe, J., Where do cyclists ride? A route choice model developed with revealed preference GPS data, https://doi.org/10.1016/j.tra.2012.07.005, Transportation Research Part A: Policy and Practice, 46(10), 1730-1740 (2012)

Bröcker, J., Korzhenevych, A., y Schürmann, C., Assesing spatial equity and efficiency impacts of transport infrastructure projects, https://doi.org/10.1016/j.trb.2009.12.008, Transportation Research Part B: Methodological, 44(7), 795-811 (2010) 
Cheng, J., Bertolini, L., y le Clercq, F., Measuring sustainable accessibility, https://doi.org/10.3141/2017-03, Transportation Research Record: Journal of the Transportation Research Board, 2017(1), 16-25 (2007)

Dijkstra, E., A note on two problems in connexion with graphs, Numerische Mathematik, 1(1), 269-271 (1959)

Farber, S., y Grandez, M., Transit accessibility, land development and socioeconomic priority: A typology of planned station catchment areas in the Greater Toronto and Hamilton Area, https://doi.org/10.5198/jtlu.2017.980, Journal of transport of Land Use, 10(1), 879-902 (2017)

Farrington, J., y Farrington, C., Rural accessibility, social inclusion and social justice: towards conceptualization, https://doi.org/10.1016/j.jtrangeo.2004.10.002, Journal of Transport Geography, 13(1), 1-12 (2005)

Frade, I., y Ribeiro, A., Bike-sharing stations: A maximal covering location approach, https://doi.org/10.1016/j.tra.2015.09.014, Transportation Research Part A: Policy and Practice, 82, $216-227$ (2015)

García, J.C., Gutiérrez, J., y Latorre, M., Optimizing the location of stations in bike-sharing programs: A GIS approach, https://doi.org/10.1016/j.apgeog.2012.07.002, Applied Geography, 35(1-2), 235-246 (2012)

Gauthie, A., Hughes, C., y otros 5 autores, Guía de planeación del sistema de bicicleta. ITDP, Institute for Transportation and Development Policy (2013)

Geurs, K. T., y Van Wee, B., Accessibility evaluation of land-use and transport strategies: review and research directions, https://doi.org/10.1016/j.jtrangeo.2003.10.005, Journal of Transport Geography, 12(2), 127-140 (2004)

Gutierrez, J., Condeco-Melhorado, A., y Martin, J.C., Using accessibility indicators and GIS to asses spatial spillovers of transport infrastructure investment, https://doi.org/10.1016/j.jtrangeo.2008.12.003, Journal of Transport Geography, 18(1), 141-152 (2010)

Handy, S.L., y Niemeier, D.A., Measuring accessibility: An exploration of issues and alternatives, https://doi.org/10.1068/a291175, Environment and Planning A: Economy and Space, 29(7), 1175-1194 (1997)

Hansen, W.G., How accessibility shapes land use, https://doi.org/10.1080/01944365908978307, Journal of the American Institute of Planners, 25(2), 73-76 (1959)

Holly, H., Metropolitan structure and the suburban hierarchy, https://doi.org/10.2307/2095909, American Sociological Review, 58(3), 417-433 (1993)

Jaramillo, C., Lizárraga, C., y Grindlay, A.L., Spatial disparity in transport social needs and public transport provision in Santiago de Cali, https://doi.org/10.1016/j.jtrangeo.2012.04.014, Journal of Transport Geography, 24, 340-357 (2012)

Jiang, H. y Levinson, D., Accessibility and the evaluation of investments on the Beijing subway, https://doi.org/10.5198/jtlu.2016.884, Journal of Transport and Land Use, 10(1), 395-408 (2016)

Kabra, A., Belavina, E., y Girotra, K., Bike-Share Systems: Accessibility and Availability, https://doi.org/10.1287/mnsc.2019.3407, Management Science, 66(9), 3799-4358 (2019)

Kotavaara, O., Antikainen, H., y Rusanen, J., Population change and accessibility by road and rail networks: GIS and statistical approach to Finland 1970-2007, https://doi.org/10.1016/j.jtrangeo.2010.10.013, Journal of Transport Geography, 19(4), 926-935 (2012)

Levinson, D. y Wu, H., Towards a general theory of access, https://doi.org/10.5198/jtlu.2020.1660, Journal of Transport and Land Use, 13(1), 129-158 (2020)

Martens, K. y Bastiaansen, J., An index to measure accessibility poverty risk. https://doi.org/10.1016/B978-0-12-8148181.00003-2. Measuring Transport Equity, 39-55, Elsevier (2019).

Papa, E., Silva, C., Brömmelstroet, M., y Hull, A., Accessibility instruments for planning practice: A review of European experiences, https://doi.org/10.5198/jtlu.2015.585, Journal of Transport and Land Use, 9(3), 57-75 (2016)

Ribeiro, A. y Silva, J., Space, development and accessibility between Portugal and Spain: the last frontier, Revista Portuguesa de Estudos Regionais, 27, 7-14 (2011)

Salonen, M., y Toivonen, T., Modelling travel time in urban networks: comparable measures for private car and public transportation, https://doi.org/10.1016/j.jtrangeo.2013.06.011, Journal of Transport Geography, 31, 143-153 (2013)

Sharma, I., Mishra, S. y otros 3 autores, Equity of transit connectivity in Tennesse cities, https://doi.org/10.1016/j.jtrangeo.2020.102750, Journal of Transport Geography, 86, 102750 (2020)

Shaheen, S.A., Guzman, S., y Zhang, H., Bikesharing in Europe, the Americas, and Asia: Past, Present, and Future, https://doi.org/10.3141/2143-20, Transportation Research Record: Journal of the Transportation Research Board, 2143(1), 159-167 (2010)

Suárez, R., Santos, D.R., y Dorta, P., Location models and GIS tools for retail site location, https://doi.org/10.1016/j.apgeog.2012.04.009, Applied Geography, 35(1-2), 12-22 (2012)

Vickerman, R., Spiekermann, K., y Wegeer, M., Accessibility and economic development in Europe, https://doi.org/10.1080/00343409950118878, Regional Studies, 33(1), 1-15 (1999)

Wang, J., Tsai, C.H., y Lin, P.C., Applying spatial-temporal analysis and retail location theory to pubic bikes site selection in Taipei, https://doi.org/10.1016/j.tra.2016.08.025, Transportation Research Part A: Policy and Practice, 94, 45-61 (2016) 